\title{
Manipulation of Preferences and Relative Utilitarianism
}

\author{
Joel Sobel ${ }^{1}$ \\ Department of Economics, University of California, \\ San Diego, La Jolla, California 92093 \\ E-mail: jsobel@ucsd.edu
}

Received July 6, 1999; published online August 8, 2001

\begin{abstract}
Given $n$ agents with who wish to divide $m$ commodities, consider the $n$-person noncooperative game with strategies consisting of concave increasing utility functions, and whose outcomes are the relative utilitarian solution. Any constrained equal-income competitive equilibrium allocation for the true utilities is shown to ba a Nash equilibrium outcome for the noncooperative game. Conditions are presented under which these are the only pure strategy equilibrium outcomes. Journal of Economic Literature Classification Numbers: C72, C78, D71. ๑ 2001 Academic Press

Key Words: utilitarian solution; bargaining; preference revelation.
\end{abstract}

\section{INTRODUCTION}

When solution concepts make predictions on the basis of unobservable information, agents will have incentives to distort their private information. This paper adds to a literature that studies the robustness of axiomatic bargaining models to strategic misrepresentation of preference information.

The paper identifies what outcomes can be expected if a social planner selects an allocation that maximizes a weighted average of the agents' reported utility functions. It studies a particular distortion game. There is a fixed quantity of a finite number of commodities. Players' strategies consist of utility functions defined over these commodities. Given the reported utility functions, the social planner provides an allocation that maximizes the weighted sum of reported preferences according to the relative utilitarian solution. (In this solution, an agent's weight is equal to the inverse of his

${ }^{1}$ I thank seminar audiences at University of California Los Angeles and Arizona State University, Ozgur Kibris, Uzi Segal, Joel Watson, an associate editor, and two referees for comments. I am also grateful to the National Science Foundation and CASBS for financial support. 
maximum utility gain.) Motivation for this solution and axiomatic derivations for it have been provided in recent work by Dhillon and Mertens (1999) and Segal (2000). If reports are limited to continuous, increasing concave functions, then any constrained equal-income competitive allocation for the true preferences (i.e., a constrained equilibrium allocation reached when agents have equal initial endowments) is an equilibrium allocation to the distortion game. This paper also provides conditions under which constrained equal-income competitive allocations are the only allocations that arise in pure-strategy equilibria of the distortion game. In equilibrium, agents report that their marginal utility is constant and equal to the price that supports the competitive equilibrium for quantities within the equal-income budget set and that their marginal utility is zero otherwise.

In earlier work, (Sobel, 1981) I studied the distortion game induced by a class of bargaining game solutions that satisfy axioms of Pareto optimality, symmetry, independence of equivalent utility representations, and midpoint domination. The first three axioms are familiar. The fourth axiom requires that the bargaining solution of an $n$-player game gives each player a utility that exceeds the disagreement utility by at least one- $n$th of the difference between his largest feasible utility and his disagreement utility. I showed that the Nash bargaining solution and the Kalai-Smorodinsky solution satisfy these four assumptions. I also showed that any constrained equal-income competitive allocation for the true utilities is an equilibrium payoff for the distortion game derived from one of these bargaining game solutions, and that if players are restricted to reporting linear strategies, then the constrained equal-income competitive allocations are the only pure-strategy Nash equilibria of the distortion game in two-player games. The relative utilitarian solution fails to satisfy the midpoint domination axiom. Nevertheless, it is still true that any constrained equal-income competitive allocation for the true utility functions is an equilibrium allocation for the distortion game. Furthermore, if players are restricted to truncated linear reports, then the only pure-strategy Nash equilibrium allocations of a modification of the distortion game are the constrained equal-income competitive allocations in two-player games.

Other authors have studied distortion games and established related results. Crawford and Varian (1979) studied the distortion game derived from the Nash bargaining solution when there is only one commodity. They showed that it is a dominant strategy to report a linear utility function. The model predicts equal division of the commodity.

Thomson $(1984,1988)$ characterized Nash equilibria for the distortion game derived from performance correspondences that yield individually rational and Pareto-efficient outcomes. Thomson (1988) found that if reported utility functions must be twice continuously differentiable and concave, and have transferable utility, then the Nash equilibria for 
the distortion game derived from the Shapley value with fixed initial endowments are exactly the constrained competitive allocations with respect to those endowments. Thomson (1984) generalized this result to an environment with transferable utility to a broader class of performance correspondences. Thomson (1987) identified a class of performance correspondences for which equilibrium outcomes of the distortion game are equal-income Walrasian equilibria for the reported preferences.

Kibris (1998) studied the distortion game derived from performance correspondences that for given initial endowments, lead to individually rational and Pareto-efficient allocations. He showed that in private good economies, constrained Walrasian allocations are equilibria to the distortion game, and that these equilibria can be supported by linear strategies. ${ }^{2}$ Because the relative utilitarian solution fails to be individually rational, Kibris's theorem does not apply in the setting of this paper. Nor does the result generalize. Equilibrium strategies will not be linear for the distortion game derived from the relative utilitarian solution.

Kurz $(1977,1980)$ studied the distortion game derived from his model of tax determination in an exchange economy. Regardless of the true preferences of the agents, the distortion game has a dominant strategy equilibrium that yields a Pareto-efficient outcome. Mas-Colell (1980) presented analogous results in his study of the manipulability of the Shapley value in an economy with an infinite number of agents of a finite number of types.

This paper provides three lessons. First, the distortion game can be viewed as another way to implement the constrained Walrasian correspondence. This point is minor, given the existence of many other procedures that do the same thing. Second, the paper generalizes the results of Sobel and Thomson. The generalization may be surprising, because in contrast to the earlier results, equilibrium strategies are necessarily nonlinear, and dominant strategy equilibria do not arise even in the one-dimensional case. The results extend the message of previous work that differences between solution concepts may disappear when one takes into account the informational advantages that bargainers have over social planners. Third, the equilibria of the distortion game predict a particular qualitative behavior for the players. In the bargaining solutions considered in this paper and earlier work, weight is given to a player's valuation of the last unit that he could receive. It is to a player's advantage to claim that his marginal unit is as valuable as possible. Because reports are limited to concave functions, equilibrium reports tend to be linear to maximize the marginal utility of marginal units. The earlier papers emphasized the importance of linear strategies. For the case of the relative utilitarian solution, it is advantageous

${ }^{2}$ Kibris (1998) provided related results for public-good environments. 
for agents to claim to be uninterested in units that they will not receive. This creates a role for truncated linear reports in which agents report that anything they receive beyond their equilibrium allocation is worthless to them.

The third lesson is the most important one. Although the assumption that the planner ignores the strategic behavior of agents when determining institutional rules is unrealistically naive, real agents are nevertheless placed in situations in which utilitarian considerations determine outcomes. Although the assumption that agents respond optimally to institutional constraints is unrealistically sophisticated, real agents do respond strategically to their environments. My analysis provides conditions under which utilitarian considerations will induce bargainers to behave in a systematic, extreme way. Bargainers, like the fox in Aesop's fable, treat what they cannot have as "sour grapes" that are not worth having while maximizing the importance of every unit that they do receive.

I should emphasize that this paper does not ask the conventional implementation question. Given a cooperative solution concept, it is customary to ask whether there exists a procedure that implements it. Miyagawa (1999) showed that a family of bargaining solutions, including the relative utilitarian solution, and solutions of Nash and Kalai-Smorodinsky, cannot be implemented in Nash equilibria because they fail the monotonicity condition shown by Maskin (1999) to be necessary for Nash implementation. Miyagawa also presented a simple mechanism that provides a subgame-perfect implementation of these solutions.

Section 2 formally describes the distortion game, and Section 3 introduces the relative utilitarian solution. Section 4 briefly describes the results for the special case of two agents and one commodity. Section 5 presents the main results. Section 6 attempts to reconcile the results with earlier findings. Section 6 introduces a one-parameter family of bargaining solutions, which includes the relative utilitarian solution, the Nash bargaining solution, and the Kalai-Smorodinsky solution. Each of these games has the property that constrained equal-income competitive allocations are equilibrium outcomes of the distortion game, but results differ as to whether equilibria are supported by linear or truncated linear strategies.

\section{DEFINITIONS AND NOTATION}

Consider $n$ agents with utility functions who are to divide a bundle of $m$ commodities. Let $\Delta_{m}$ denote the $m-1$ dimensional simplex and let $e_{k} \in \Delta_{m}$ be the unit vector in the $k$ th direction. Let $\mathbf{a} \equiv(a, \ldots, a)$. An outcome will be an element of the set

$$
T=\left\{x=\left(x_{1}, \ldots, x_{n}\right) \in \mathbf{R}^{n m}: x_{i} \in \mathbf{R}^{m} \text { for } i=1, \ldots, n, \text { and } \mathbf{0} \leq \sum_{i=1}^{n} x_{i} \leq \mathbf{1}\right\},
$$


where agent $i$ receives $x_{i}$. The true utility function of player $i$ is denoted by $u_{i}$. These functions are assumed to be concave and strictly increasing. The players report utilities that are restricted to lie in the class $U$, where $\mathcal{U}$ comprises those functions $U: \mathbf{R}^{m} \rightarrow[0,1]$ such that (i) $U$ is continuous, increasing, and concave in $\mathbf{R}^{m}$ and (ii) $U$ is normalized so that $U(\mathbf{0})=0$ and $U(\mathbf{1})=1$. The class of admissible utilities should include those functions that are credible representations of the agents' true preferences. Condition (i) is a regularity assumption on the range of potential players. The concavity assumption means that the agents cannot pretend to have increasing marginal utility. In Section 4 I discuss the implications of relaxing the assumption. To guarantee existence of equilibrium, $\mathcal{U}$ must be permitted to include functions that are not strictly increasing. Since the solution to the bargaining problem studied in this paper is independent of affine transformations, condition (ii) is not essential.

The distortion game is played by each agent revealing a utility function in $U$. Let $U_{i}$ denote the utility function revealed by player $i$. Given the report $U=\left(U_{1}, \ldots, U_{n}\right)$, a set of outcomes $B(U)$ is selected. $B(U)$ is the set of allocations that give rise to a bargaining solution determined by utility functions $U$. In Section 3 I discuss the particular bargaining solution that is the focus of this paper. However, to define the distortion game, it is only necessary that $B(U)$ be a nonempty subset of $T$ for any admissible reported utilities.

If $B(U)$ is single-valued, then all elements of the definition of the distortion game would be in place. Players would be restricted to admissible strategies and, denoting the $i$ th component of $B(U)$ by $B_{i}(U)$, the payoff to player $i$ would be $u_{i}\left(B_{i}(U)\right)$. Although it is typical to assume that the bargaining solution selects a unique utility for each player, there is no reason to believe that there will be a unique allocation associated with these utilities. $B(U)$ typically will not be single valued, so it is necessary to specify how to make a selection from the bargaining solution. I assume that agents have the right to select their most preferred outcome in $B(\cdot)$. This assumption leads to the following definition.

DEFINITION 2.1. $\left(U^{*} ; x^{*}\right)=\left(\left(U_{1}^{*}, \ldots, U_{n}^{*}\right) ;\left(x_{1}^{*}, \ldots, x_{n}^{*}\right)\right)$ is an equilibrium for the distortion game determined by $B$ if and only if $x^{*} \in B\left(U^{*}\right)$ and for each $i,\left(U_{i}^{*}, x_{i}^{*}\right)$ solves $\max u_{i}\left(x_{i}\right)$ subject to $x_{i} \in\left\{B_{i}\left(U_{i}, U_{-i}^{*}\right): U_{i} \in \mathcal{U}\right\}$.

The definition implies that for equilibrium reports $U^{*}$, there exists an allocation $x^{*} \in B\left(U^{*}\right)$ such that $x_{i}^{*}$ solves max $u_{i}\left(x_{i}\right)$ subject to $x_{i} \in B_{i}\left(U^{*}\right)$. That is, players' true preferences can agree on a most preferred allocation in $B\left(U^{*}\right)$. Implicit in the definition is the assumption that players have complete information. In particular, players must know their opponents' true utility function. 
By expanding the strategy sets of the agents, it is possible to show that the assumption that agents can select their most preferred outcome in $B(U)$ can be made without loss of generality. In earlier work (Sobel, 1981), my strategy sets of the distortion game consisted of both reported preferences and tie-breaking rules that make unique selections from the correspondence $B$. That paper introduced a class of tie-breaking rules, essentially possible allocations, and showed that in equilibrium agents will select their most preferred outcome in $B$. An equilibrium for the distortion game corresponds to a Nash equilibrium of an extended game in which each agent reports a tie-breaking rule as well as a utility function. Consequently, there is no loss in generality (and some gain in simplicity) in using Definition 2.1. ${ }^{3}$ Thomson (1984) adopted this definition.

Bargaining solutions uniquely determine the utilities of agents according to the agents' reported preferences. One might think that players would be indifferent between all of the allocations in $B(U)$. However, unless an agent reports his true utility function, there is no reason to expect that he would be indifferent over all elements in the bargaining solution according to his true preferences. In the equilibria described in Section $5, B(U)$ consists of a budget hyperplane, and agents generally will not be indifferent over the elements in $B$.

In what follows, I prove that the Nash equilibrium outcomes in the distortion game correspond to certain competitive outcomes.

DEFINITION 2.2. A constrained equal-income competitive equilibrium (EICE) is a pair, $\left(p^{*}, x^{*}\right)$, where $p^{*} \in \Delta_{m}, x^{*} \in T$, and $x_{i}^{*}$ solves

$$
\max u_{i}\left(x_{i}\right) \text { subject to } p^{*} \cdot x_{i} \leq \frac{1}{n} p^{*} \cdot \mathbf{1} \text { and } \mathbf{0} \leq x_{i} \leq \mathbf{1} .
$$

In an exchange economy, an equal-income competitive equilibrium is a competitive equilibrium to the economy in which agents have equal initial endowments. In a constrained equal-income competitive equilibrium, agents' demands are restricted to be both in their budget set and feasible relative to the total resources of the economy. The constraint that agent $i$ 's demand $x_{i}$ satisfy $\mathbf{0} \leq x_{i} \leq \mathbf{1}$ is not placed on (unconstrained) equalincome competitive equilibria. ${ }^{4}$ Any equal-income competitive equilibrium is a constrained equal-income competitive equilibrium, and, provided that

${ }^{3}$ To interpret the results of this paper as implementation theorems, one should return to the formulation in Sobel (1981) and assume that the strategies in the distortion game are both utility functions and tie-breaking rules.

${ }^{4}$ Hurwicz, Maskin, and Postlewaite (1995) introduced the concept of constrained competitive equilibrium. This is the smallest extension of the competitive correspondence that can be implemented in Nash strategies. 
preferences are convex, any interior constrained equal-income competitive equilibrium is an equal-income competitive equilibrium. I call $x^{*}$ in the definition of EICE a competitive allocation. I concentrate on the constrained competitive allocations rather than the unconstrained allocations, because the former set turns out to be the one related to the equilibrium outcomes of distortion games.

\section{THE RELATIVE UTILITARIAN SOLUTION}

This paper studies the distortion game induced by the relative utilitarian solution proposed by Cao (1982), Dhillon and Mertens (1999), Karni and Safra (1996), Karni (1998), and Segal (2000). Cao's approach focused on a bargaining problem. The other authors presented the functional form that I describe later for social-choice problems. This section presents additional detail on bargaining games. The discussion in this section is a bit more general than needed for the main results in Section 5. I include the additional detail to contrast this paper with earlier work and analyze the more general games in Section 6.

A bargaining game is described by a pair $(S, d)$, where, (i) $d=\left(d_{1}, \ldots\right.$, $\left.d_{n}\right) \in \mathbf{R}^{n}$ and (ii) $S \subset \mathbf{R}^{n}$ is compact, convex, and contains $d$ as well as some point $s>d$.

The set $S$ is normally interpreted as the set of feasible utility payoffs to the players. A point $s \in S$ can be achieved if all players agree to it. In case of agreement, player $i$ receives $s_{i}$. If the players are unable to agree, then the outcome $d$, called the disagreement outcome, is the result.

In this paper the reported utilities $U=\left(U_{1}, \ldots, U_{n}\right)$ with each $U_{i} \in \mathscr{U}$ determine the set $S$. That is,

$$
S=S(U)=\left\{s=\left(s_{1}, \ldots, s_{n}\right): s_{i}=U_{i}\left(x_{i}\right) \text { for some } x \in T\right\} .
$$

The disagreement outcome will always be taken to be $\mathbf{0}=U(\mathbf{0})$.

When the functions $U_{i}$ are admissible, the set $S$ is compact and convex, and contains a point $s>\mathbf{0}$. In fact, the $i$ th unit vector $e_{i} \in S$ for $i=1, \ldots, n$ and $\Delta_{n} \subset S$.

Beginning with Nash (1950), axiomatic bargaining theory has focused on solutions to bargaining games. A quasi-solution is a nonempty-valued correspondence $f$, defined on the class of all bargaining games with $f(S, d)=\left(f_{1}(S, d), \ldots, f_{n}(S, d)\right) \subset S$ for all pairs $(S, d) . f$ is a solution if it is single valued. Nash characterized his bargaining solution in terms of four axioms, three of which are relevant here.

Axiom 1 (Pareto efficiency). If $f(S, d)=x$ and $y \geq x$, then either $y=x$ or $y \notin S$. 
Axiom 2 (independence of equivalent utility representations). If $(S, d)$ and $\left(S^{\prime}, d^{\prime}\right)$ are bargaining games such that

$$
S^{\prime}=\left\{s^{\prime}=\left(s_{1}^{\prime}, \ldots, s_{n}^{\prime}\right): s_{i}^{\prime}=a_{i} s_{i}+b_{i} \text { for } s=\left(s_{1}, \ldots, s_{n}\right) \in S\right\}
$$

and

$$
d^{\prime}=\left(a_{1} d_{1}+b_{1}, \ldots, a_{n} d_{n}+b_{n}\right),
$$

where $a_{i}>0$ for all $i$, then

$$
f\left(S^{\prime}, d^{\prime}\right)=\left(a_{1} f_{1}(S, d)+b_{1}, \ldots, a_{n} f_{n}(S, d)+b_{n}\right) .
$$

Axiom 3 (symmetry). If $(S, d)$ is a symmetric game [i.e., $s=\left(s_{1}, \ldots, s_{n}\right)$ $\in S$ if and only if $\left(s_{\pi(1)}, \ldots, s_{\pi(n)}\right) \in S$ for any permutation $\left.\pi\right]$, then $f_{i}(S, d)$ is independent of $i$.

Given a bargaining game $(S, d)$, let $\bar{s}_{i} \equiv \max \left\{s_{i}: s=\left(s_{1}, \ldots, s_{n}\right): s \in S\right\}$ be player $i$ 's maximum utility. The relative utilitarian quasi-solution consists!of

$$
\begin{array}{r}
s^{*}=\left(s_{1}^{*}, \ldots, s_{n}^{*}\right): s^{*} \text { solves } \max \sum_{i=1}^{n}\left(\frac{s_{i}}{\bar{s}_{i}-d_{i}}\right) \text { subject to } \\
\left.s=\left(s_{1}, \ldots, s_{n}\right) \in S\right\} .
\end{array}
$$

Because (1) may contain more than one point, it defines a quasi-solution rather than a solution. It is apparent that the relative utilitarian quasisolution is invariant with respect to equivalent utility representations and is Pareto efficient with respect to the reported utilities.

I say that a game is $0-1$ normalized if $d=0$ and $\bar{s}_{i}=1$ for $i=1, \ldots, n .^{5}$ A bargaining set obtained when players report utility functions in the class $\mathcal{U}$ must be $0-1$ normalized. Note that if $(S, d)$ is $0-1$ normalized, then (1) simplifies to

$$
\begin{array}{r}
\left\{s^{*}=\left(s_{1}^{*}, \ldots, s_{n}^{*}\right): s^{*} \text { solves } \max \sum_{i=1}^{n} s_{i}\right. \text { subject to } \\
\left.s=\left(s_{1}, \ldots, s_{n}\right) \in S\right\} .
\end{array}
$$

One forms a relative-utilitarian solution by making a selection from (1). Working with symmetric relative-utilitarian solutions is convenient. Thus in what follows, assume that the social planner makes a selection from (1)

${ }^{5}$ The relative utilitarian quasi-solution is invariant with respect to equivalent utility representations. Any game can be taken to be $0-1$ normalized without loss of generality by taking a positive affine transformation of utility functions that maps agent $i$ 's disagreement payoff to 0 and agent $i$ 's maximum payoff to 1 . 
whenever (1) contains more than one point. The selection can be arbitrary, although when $S$ is symmetric, it makes sense to assume that the selection is symmetric. Denote the selection by $R I(S)$. Any symmetric relative-utilitarian solution plainly satisfies the symmetry axiom.

Admissible reported utilities $U=\left(U_{1}, \ldots, U_{n}\right)$ determine a set $S(U)$ of feasible utilities with respect to the reported preferences. The social planner will select an allocation according to the relative utilitarian solution. This selection determines the set $B(U)$ of resource allocations that give rise to the relative utilitarian solution,

$$
B(U)=\left\{x=\left(x_{1}, \ldots, x_{n}\right) \in T:\left(U_{1}\left(x_{1}\right), \ldots, U_{n}\left(x_{n}\right)\right)=R I(S(U))\right\} .
$$

It follows that $x^{*} \in B(U)$ if and only if $x^{*}$ solves

$$
\max \sum_{i=1}^{n} U_{i}\left(x_{i}\right) \text { subject to } x=\left(x_{1}, \ldots, x_{n}\right) \in T \text {. }
$$

In the equilibria described in Sections 4 and 5, agents report utility functions that are not necessarily strictly increasing. This creates the possibility that there exist $x$ and $y \in B(U)$ such that $\sum_{i=1}^{n} y_{i}<\sum_{i=1}^{n} x_{i}$; that is, both $x$ and $y$ solve the planner's optimization problem, but $y$ does so using strictly less of each commodity. When this situation arises, the social planner might restrict attention to allocations that are resource conserving,

$$
B^{*}(U)=\left\{x \in B(U): \text { if } \sum_{i=1}^{n} y_{i} \leq \sum_{i=1}^{n} x_{i}, \text { then } y \notin B(U) \text { or } y=x\right\} .
$$

If all of the reported utilities are strictly increasing, then $B^{*}(U)=B(U)$. In general, $B^{*}(U) \subseteq B(U) . B^{*}(U)$ comprises the allocations that give rise to utilities that solve the social planner's problem without wasting resources. The distortion game determined by a relative utilitarian solution $B(\cdot)$ is called the relative utilitarian distortion game. The corresponding game determined by $B^{*}(U)$ is called the resource-conserving relative utilitarian distortion game.

Sobel (1981) described equilibria of distortion games for bargaining solutions that satisfy Axioms 1, 2, and 3, as well as Axiom 4.

Axiom 4 (midpoint domination). $\quad f_{i}(S, d) \geq \frac{1}{n\left(\bar{s}_{i}-d_{i}\right)}$ for all $i$.

If $(S, d)$ is $0-1$ normalized, then Axiom 4 becomes $f_{i}(S, d) \geq 1 / n$ for all $i$. I (Sobel, 1981) introduced Axiom 4 under the name symmetric monotonicity. Thomson (1994) called it "midpoint domination."

I also demonstrated that any bargaining solution for a two-person game that is risk dominant (see Roth, 1979) satisfies Axiom 4. It follows from Roth (1979) that the Nash and Kalai-Smorodinsky bargaining solutions 
satisfy Axiom 4. On the other hand, the relative utilitarian solution does not satisfy midpoint domination. For instance, let

$$
S=\left\{s=\left(s_{1}, s_{2}\right): s_{1} \in[0,1] \text { and } s_{2} \in\left[0,1-a s_{1}\right]\right\} .
$$

When $a \in(0,1)$ the solution to max $s_{1}+s_{2}$ subject to $\left(s_{1}, s_{2}\right) \in S$ is $(1,1-a)$. For $a>0.5$, player 2 receives utility less than 0.5 .

The example demonstrates that my earlier theorem (Sobel, 1981) does not apply to the relative utilitarian solution. Nevertheless, as I now demonstrate, the qualitative conclusions of the earlier work do apply.

\section{THE ONE-COMMODITY CASE}

To get an intuition for the results, consider the special case with two players and one commodity. With only one commodity, all strictly monotonic preferences are ordinally equivalent. Given the symmetry of the game, one would expect equal division to be the only equilibrium outcome of the distortion game. This conjecture is correct. Each agent can guarantee at least one-half of the commodity by reporting the utility function $U^{*}(x)=$ $\min [2 x, 1]$. This utility function is concave, continuous, and increasing. Furthermore, a player who reports this utility must receive at least one-half. To see this, note that under $U^{*}(\cdot)$, the agent asserts that the marginal utility of each $x<0.5$ is 2 . If the solution is to give the other player more than onehalf, then the other player's reported marginal utility must be greater than or equal to 2 for each of the first $1-x>0.5$ units. This is not consistent with monotonicity and the restriction that $U(1)=1$. Because each player can guarantee at least half of the commodity, the equilibrium must give each player at least half. Hence in equilibrium, both agents must receive exactly half.

The one-commodity case provides several lessons. First, the outcome of the game is efficient with respect to the true preferences. (Indeed, it is the unique EICE.) Second, equilibrium strategies of the agents are not linear, and the equilibrium is not a dominant strategy equilibrium. Note that if one agent reported the linear utility function $U(x)=x$, then for any $\epsilon>0$ the opponent could respond with the function $V(x)=\min [(1+\epsilon) x, 1]$ and obtain almost the entire unit of the commodity (the share $1 /(1+\epsilon)$ ). Third, the equilibrium strategies are not strictly increasing. Equilibria would not exist if players were restricted to strictly increasing strategies. Agents could guarantee arbitrarily close to one-half by playing continuous approximations to $U^{*}(\cdot)$. Hence in equilibrium, both players must receive one-half. However, given any continuous strategy of one player, it is possible for the other player to obtain strictly more than one-half by choosing a better approximation to $U^{*}(\cdot)$. Fourth, although the equilibrium strategies are not 
linear, they are linear when restricted to $x \leq 0.5$, the quantities that the agent consumes in equilibrium. When making a claim for a marginal unit, it is in the bargainers' interest to claim that the marginal unit is as valuable as possible to them. They deny any interest in units that they are not going to receive and report nondecreasing marginal utility over the units that they do receive. These observations generalize to the case of more than one commodity.

Although agents lack strictly dominant strategies in the one-commodity model, when players are restricted to truncated linear strategies, the equilibrium strategies are the only strategies that satisfy iterated deletion of weakly dominated strategies, provided that the selection from the relative utilitarian correspondence is symmetric. Limit players to strategies in the set $\mathscr{L}=\{U(x)=\min [a x, 1]$ for $a \geq 1\}$. This restriction is plausible because given any $U \in \mathscr{U}$, a player always has a best response in $\mathscr{L}$. Let $X_{b}=\{\min [a x, 1] \in \mathscr{L}: 2 \geq a \geq b\}$. Suppose that agent $i$ uses strategies in $X_{b}$. Player $j \neq i$ receives less than one-half when he uses $\min [a x, 1]$ for $a<b$ or $a>2$. If $a=b$, then the player receives exactly one-half when the other player uses the same strategy (by symmetry) and strictly less against any other strategy in $X_{b}$. On the other hand, the strategy $U^{*}$ guarantees that agent $j$ receives $x=0.5$. Consequently, $U^{*}(\cdot)$ is the only strategy that survives iterative deletion of weakly dominated strategies. The same argument applies when there are $n>2$ players; each agent uses the function $\min [n x, 1]$ in this case.

The one-commodity case also provides a clear indication of the importance of the assumption that agents' reports are concave functions. Assume instead that agents could report arbitrary monotonic functions and that agent 1 reports $[x]$, where $[\cdot]$ is the greatest integer function. ${ }^{6}$ A solution to the problem

$$
\max [x]+V(1-x) \text { subject to } x \in[0,1]
$$

is to give agent 1 everything for all $V \in \mathcal{U}$. (Given agent 1's report, the value of the objective function in (4) can be no greater than 1; this upper bound is attained if player 1 receives everything.) An equilibrium in which all players report "all-or-nothing" preferences exists whenever arbitrary monotonic reports are permitted. ${ }^{7}$

${ }^{6}$ The greatest integer function is not continuous. Best replies generally would not exist if agents could report arbitrary (nonconcave) continuous utility functions. Continuous approximations to [·] would be approximate best replies.

${ }^{7}$ When reports do not need to be concave, it is natural to permit the bargaining solution to randomize. Symmetry suggests that the solution to the bargaining problem when all agents report "all-or-nothing" preferences is to give everything to a randomly selected player. 


\section{THE GENERAL CASE}

When more than one commodity exists, agents may not have the same ordinal preferences. It may not be efficient to split each unit equally. In this section I demonstrate that the equilibrium outcomes of the distortion game are still efficient in the general setting.

For $p \in \Delta_{m}$, let $L(z ; p) \equiv \min [n p \cdot z, 1]$. Given $V \in \mathcal{U}$,

$$
\lim _{h \rightarrow 0^{+}}\left[V(z)-V\left(z-h e_{k}\right)\right] / h
$$

exists; let $D_{k}^{-} V(z)$ denote the limit. When $V(\cdot)$ is differentiable at $x, D_{k}(z)$ is a partial derivative, generally a one-sided partial derivative. Let $\nabla V(z)=$ $\left(D_{1}^{-}(z), \ldots, D_{m}^{-}(z)\right)$. Because $V(\mathbf{0})=0$ and $V$ is concave,

$$
V(z) \geq \nabla V(z) \cdot z \text { for all } z \geq \mathbf{0} .
$$

LEMMA 5.1. If player $j$ reports the truncated utility $L(\cdot ; p)$, then for all admissible $U_{-j}$, if $x=\left(x_{1}, \ldots, x_{n}\right) \in B\left(L(\cdot ; p), U_{-j}\right)$, then $n p \cdot x_{j} \geq 1$.

Lemma 5.1 is a special case of Lemma 6.1, which I state and prove in Section 6. The lemma establishes that using a truncated linear report, a player can guarantee an allocation that is at least as good as equal division according to the reported preferences. If players are restricted to reported preferences of the form $L(z ; p)$ for $p \in \Delta_{m}$, then the result is particularly easy to understand. In this case, equal division yields the maximum value of the relative-utilitarian social welfare function. Hence any allocation in $B(\cdot)$ for truncated linear reports must give each agent utility 1 with respect to his reported preference. This is the conclusion of the lemma. The actual proof is a bit more involved, because agents are not limited to truncated linear reports.

THEOREM 5.1. If $\left(p^{*}, x^{*}\right)$ is an EICE for the true preferences, then $\left(L\left(\cdot ; p^{*}\right), \ldots, L\left(\cdot ; p^{*}\right) ; x^{*}\right)$ is a Nash equilibrium for the relative utilitarian distortion game.

Proof. Assume that all players except player $i$ use the strategy $L\left(\cdot ; p^{*}\right)$. Assume that player $i$ selects the strategy $V$. Denote the resulting allocation by $x=\left(x_{1}, \ldots, x_{n}\right)$. It follows from Lemma 5.1 that for all $i \neq j, n p^{*}$. $x_{j} \geq 1$. Because $x \in T, n p^{*} \cdot x_{i} \leq 1$. It follows that the maximum utility that player $i$ could receive in response to his opponents' strategies is

$$
\max u_{i}\left(x_{i}\right) \text { subject to } n p^{*} \cdot x_{i} \leq 1 \text { and } \mathbf{0} \leq x_{i} \leq \mathbf{1} .
$$

Because $\left(p^{*}, x^{*}\right)$ is an EICE, $x_{i}^{*}$ solves (6). Because

$$
x^{*} \in B\left(L\left(\cdot ; p^{*}\right), \ldots, L\left(\cdot ; p^{*}\right)\right)
$$

it follows that this is a Nash equilibrium for player $i$ to report $L\left(\cdot, p^{*}\right)$ and obtain the EICE allocation. 
The theorem demonstrates that any EICE allocation is a Nash equilibrium of the distortion game. The lemma guarantees that if each agent reports the same truncated linear strategy $L\left(\cdot, p^{*}\right)$, then the set of solutions to the planner's problem comprises the entire budget hyperplane with normal $p^{*}$ through equal division. If $p^{*}$ supports an EICE, then all players can agree on a most preferred outcome in the set of allocations that maximize the relative utilitarian objective function. Consequently, an EICE allocation is an equilibrium of the distortion game.

A curious aspect of the equilibrium strategies supports an EICE allocation. The value of the social planner's optimization problem is equal to $n$, the maximum feasible value. Even though there is conflict over the goods, the equilibrium allocation provides each agent utility 1 (according to reported preferences). If the social planner were naive enough to believe that the reported preferences were honest representations of the agent's motives, then he or she would be delighted to know that every agent received his maximum utility.

In the one-commodity case, the equilibrium allocation was unique. Because there may be multiple ECIE allocations, the uniqueness property will not carry over into the many-commodity case. The best that one might hope for is that the only equilibrium allocations are the EICE allocations. This result fails to hold, as the next example demonstrates. The example is a modification of an example that I gave in earlier work (Sobel, 1981).

EXAMPLE 5.1. In a two-consumer, two-commodity model, let $u_{1}\left(x_{1}, x_{2}\right)$ $=x_{1}^{5 / 6} x_{2}^{1 / 6}, u_{2}\left(x_{1}, x_{2}\right)=x_{1}^{1 / 2} x_{2}^{1 / 2}, U_{1}\left(x_{1}, x_{2}\right)=\min \left[\left(5 x_{1}+3 x_{2}\right) / 4,1\right]$, and $U_{2}\left(x_{1}, x_{2}\right)=x_{1}^{1 / 2} x_{2}^{1 / 2}$. For $i=1$ and $2, u_{i}$ and $U_{i} \in U$. Routine computation shows that $\left(U_{1}, U_{2}, x^{*}\right)$ is a Nash equilibrium for the distortion game. The allocation $x^{*}$ gives agent $1(3 / 5,1 / 3)$ and agent 2 $(2 / 5,2 / 3)$. In this example, the unique EICE for the true preferences is $\left(p^{*}, y^{*}\right)=((2 / 3,1 / 3),(5 / 8,1 / 4))$ [Here $y^{*}$ is player 1's allocation in the equilibrium. Player 2 receives $\left.1-y^{*}=(3 / 8,3 / 4)\right]$. Computation shows that the player 1 prefers the inefficient outcome $x^{*}$ to the EICE. Also, player 2 is worse off at the Nash equilibrium even though he is reporting his true utility function.

In earlier work (Sobel, 1981) I showed that in two-player games, the only equilibrium allocations of distortion games derived from bargaining solutions satisfying Axioms 1-4 are EICE allocations provided that agents are restricted to linear strategies. This restriction makes no sense in the present context, because linear strategies do not support the equilibria described in Theorem 5.1. It does suggest that a partial converse to Theorem 5.2 holds if players are limited to truncated linear strategies. Even this result fails to hold, as the next example demonstrates. 
EXAMPLE 5.2. In a two-consumer, two-commodity model, let $u_{1}\left(x_{11}, x_{12}\right)$ $=x_{11}^{2 / 3} x_{12}^{1 / 3}, u_{2}\left(x_{21}, x_{22}\right)=x_{21}^{1 / 3} x_{22}^{2 / 3}, U_{1}\left(x_{11}, x_{12}\right)=\min \left[2\left(x_{11}+2 x_{12}\right) / 3,1\right]$, and $U_{2}\left(x_{21}, x_{22}\right)=\min \left[2\left(2 x_{21}+x_{22}\right) / 3,1\right]$. For $i=1$ and $2, u_{i} \in \mathcal{U}$. Let the allocation $x^{*}$ give both agents $(1 / 2,1 / 2)$. A computation shows that $\left(U_{1}, U_{2}, x^{*}\right)$ is a Nash equilibrium for the distortion game. Lemma 5.1 implies that an upper bound to the utility that agent 1 can attain is the value of $\max u_{1}\left(x_{11}, x_{12}\right)$ subject to $2\left(2 x_{11}+x_{12}\right) / 3 \leq 1$ [because the lemma guarantees that player 2 's allocation satisfies $2\left(2_{21}+x_{22}\right) / 3 \geq 1$ ] and similarly that the upper bound for player 2 is the value of $\max u_{2}\left(x_{21}, x_{22}\right)$ subject to $2\left(x_{21}+2 x_{22}\right) / 3 \leq 1$. For the reported preferences $\left(U_{1}, U_{2}\right)$,

$$
\begin{array}{r}
B\left(U_{1}, U_{2}\right)=\left\{\left(x_{11}, x_{12}, x_{21}, x_{22}\right) \in T: 2\left(x_{11}+2 x_{12}\right) / 3 \geq 1\right. \text { and } \\
\left.2\left(2 x_{21}+x_{22}\right) / 3 \geq 1\right\} .
\end{array}
$$

For the true preferences, both agents agree that $x^{*}$ is the best allocation in $B\left(U_{1}, U_{2}\right)$. It is apparent that the allocation $x^{*}$ is not efficient. The only EICE in this example is $p=(1 / 2,1 / 2),\left(x_{11}, x_{12}\right)=(2 / 3,1 / 3)$ and $\left(x_{21}, x_{22}\right)=(1 / 3,2 / 3)$.

In the example, $B(U)$ contains allocations that are not resource conserving. All of the endowment need not be used to attain the maximum in the social planner's utility function. This suggests the possibility that the inefficient equilibrium would disappear if the planner used a resource-conserving selection from the set of maximizing allocations.

Imposing the additional requirement that the solution be resource conserving shrinks the set of allocations. The planner allocates the minimum quantity of resources needed to maximize the relative utilitarian objective function. For the reported preferences

$$
\begin{aligned}
B^{*}\left(U_{1}, U_{2}\right) & =\left\{\left(0, \frac{3}{4}, s, \frac{3}{2}-2 s\right), s \in\left(\frac{5}{8}, \frac{3}{4}\right)\right\} \\
& \cup\left\{\left(\frac{3}{2}-2 t, t, \frac{3}{4}, 0\right), t \in\left(\frac{5}{8}, \frac{3}{4}\right)\right\},
\end{aligned}
$$

$x^{*} \notin B^{*}\left(U_{1}, U_{2}\right)$, and the agents have conflicting preferences over allocations in $B^{*}\left(U_{1}, U_{2}\right)$. Agent 1 prefers the allocation $\left(x_{11}, x_{12}\right)=(1 / 4,5 / 8)$ and $\left(x_{21}, x_{22}\right)=(3 / 4,0)$, whereas agent 2 prefers the allocation $\left(x_{11}, x_{12}\right)=$ $(0,3 / 4)$ and $\left(x_{21}, x_{22}\right)=(5 / 8,1 / 4)$.

The two examples suggest that a partial converse to Theorem 5.1 requires both a restriction on strategies and a selection from the set of maximizing allocations. The following theorems provide the results.

THEOREM 5.2. Let $n=2$. If $\left(L\left(\cdot ; p_{1}\right), L\left(\cdot ; p_{2}\right) ; x^{*}\right)$ is a Nash equilibrium for the resource-conserving relative-utilitarian distortion game, then $x^{*}$ is an EICE allocation. 
The idea of the proof is to show that if both players use truncated linear strategies, then they must both use the same truncated linear strategy. From this observation, it is clear that outcome must be an EICE.

Proof. It follows from Lemma 5.1 that for all $i, 2 p_{i} \cdot x_{i}^{*} \geq 1$. Let $j \neq i$. Because $x^{*} \in T, 2 p_{j} \cdot x_{i}^{*} \leq 1$. It follows that the maximum utility that player $i$ could receive in response to his opponent's strategy is

$$
\max u_{i}\left(x_{i}\right) \text { subject to } 2 p_{j} \cdot x_{i} \leq 1 \text { and } \mathbf{0} \leq x_{i} \leq \mathbf{1} .
$$

Player $i$ can indeed achieve this payoff by imitating player $j$ and using the strategy $L\left(\cdot ; p_{j}\right)$. It follows from monotonicity of $u_{i}(\cdot)$ that $2 p_{j} \cdot x_{i}^{*}=1$. Thus the resource-conserving condition implies that $p_{i}=p_{j}$, for otherwise the planner would solve (3) with an allocation $x^{*}$ such that $2 p_{j} \cdot x_{i}^{*}<1$. It follows that in equilibrium, $p_{i}=p_{j}$. Consequently, (7) implies that $x^{*}$ is an EICE allocation.

I conjecture that Theorem 5.2 holds when $n>2$. Kibris (1998) demonstrated that Theorem 5.2 would hold for $n \geq 2$ if players' true utility functions satisfied a boundary condition (so that indifference curves do not intersect the boundary of the Edgeworth box).

There are two justifications for the restriction to truncated linear strategies. From the stand-point of implementation, the planner can make whatever restrictions on strategies needed to guarantee desirable outcomes. From the stand-point of the agents' strategic behavior, it is possible to show that even when there are no restrictions on admissible reports (other than continuity, monotonicity, and concavity), agents always have a truncated linear best response.

\section{RECONCILIATION}

Earlier results demonstrate that the Nash equilibria of distortion games derived from admissible bargaining games are EICE allocations and that in these equilibria, players report linear utility functions. This paper studies the distortion game derived from a bargaining game that fails to satisfy the assumption of midpoint domination. Nonetheless, the Nash equilibria of distortion games are EICE allocations. Equilibrium reports are nonlinear but are piecewise linear. There is no logical contradiction here. The relative utilitarian solution does not satisfy my assumptions in the earlier paper (Sobel, 1981).

It may be instructive to reflect more on the sense in which the current results generalize the earlier results. First, the restriction to linear strategies in the earlier work is not always necessary. For the distortion game derived from the Nash bargaining solution, one can support the same outcomes 
as equilibria by using piecewise linear reports of the kind introduced in this paper. Second, although the relative utilitarian solution fails midpoint domination, it does not fail midpoint domination if players are restricted to truncated linear strategies that are used to support equilibria. This is essentially the conclusion of Lemma 5.1.

To make these points formally, consider a family of bargaining solutions. For $s=\left(s_{1}, \ldots, s_{n}\right)$ and $a \leq 1$, let $W(s, a)=\left\{\sum_{i=1}^{n} 1 / n s_{i}^{a}\right\}^{1 / a} \cdot{ }^{8}$ Let $(S, d)$ be $0-1$ normalized. For $a<1, W(\cdot, a)$ is strictly concave and generates a bargaining solution

$$
f(S ; a)=\left\{s^{*}=\left(s_{1}^{*}, \ldots, s_{n}^{*}\right): s^{*} \text { solves } \max W(s, a) \text { subject to } s \in S\right\} .
$$

Of course, $W(s, 1)$ is the utilitarian objective function. Because $W(s, 0)=$ $\left\{\prod_{i=1}^{n} s_{i}\right\}^{1 / n}$ and $\lim _{a \rightarrow-\infty} W(s, a)=\min \left\{s_{1}, \ldots, s_{n}\right\}, f(S ; 0)$ corresponds to the Nash bargaining solution and $f(S ;-\infty)$ corresponds to the KalaiSmorodinsky solution. Increases in $a$ tend to make $W(\cdot)$ more like the utilitarian solution, whereas decreases in $a$ make the solution more sensitive to the relative welfare of agent who receives the lowest (normalized) utility.

Because the family of bargaining solutions is defined as solutions to optimization problems, one might be tempted to conclude that they all satisfy Nash's independence of irrelevant alternatives axiom. In fact, only $f(S ; 0)$ (the Nash bargaining solution) satisfies this axiom. The possible confusion arises because I have defined the solution for $0-1$ normalized bargaining games. In general, changing the maximum utility available to a bargainer will change that bargainer's weight in $W(\cdot)$, leading to the possible violation of the independence of irrelevant alternatives axiom.

Imagine a family of distortion games that varies with the parameter $a$. For $a \leq 0$, one can show that the bargaining solutions satisfy the midpoint domination axiom. Hence earlier results (Sobel, 1981) can be used to characterize the solution to the distortion game. For $a \geq 0$, Lemma 5.1 continues to hold. Hence the results from the previous section apply. In all cases, EICE allocations are equilibria of the distortion game. In the case of solutions with nonnegative parameter $a$, these equilibria are supported by truncated linear strategies. When $a$ is nonpositive, the equilibrium is supported by linear strategies. Curiously, the Nash bargaining solution is the only one in which both types of strategy support the equilibrium.

LEMMA 6.1. If player $j$ reports the truncated utility $L(\cdot ; p)$, then for all admissible $U_{-j}$, if $x=\left(x_{1}, \ldots, x_{n}\right) \in B\left(L(\cdot ; p), U_{-j} ; a\right)$ and $a>0$, then $n p \cdot x_{j} \geq 1$.

${ }^{8}$ When $a=0$, define $W(s, 0)=\lim _{a \rightarrow 0} W(s, a)=\left\{\Pi_{i=1}^{n} s_{i}\right\}^{\frac{1}{n}}$. 
Proof. Assume that player $j$ uses the strategy $L(\cdot ; p)$. Suppose that if player $i$ selected the strategy $V$, then the allocation would be $x=\left(x_{1}, \ldots, x_{n}\right)$. To obtain a contradiction, assume that

$$
n p \cdot x_{j}<1 \text {. }
$$

Because $x$ must maximize

$$
\max \sum_{i=1}^{n}\left[U_{i}\left(x_{i}\right)\right]^{a} \text { subject to } x=\left(x_{1}, \ldots, x_{n}\right) \in T,
$$

for the reported preferences,

$$
\left[n p \cdot\left(x_{j}+h\right)\right]^{a}+\left[V\left(x_{i}-h\right)\right]^{a} \leq\left[n p \cdot x_{j}\right]^{a}+\left[V\left(x_{i}\right)\right]^{a}
$$

for all $\mathbf{0} \leq h \leq x_{i}$. By (9), the partial derivative of $\left[n p \cdot x_{j}\right]^{a}+\left[V\left(x_{i}\right)\right]^{a}$ in the direction of $e_{k}$ must be less than or equal to 0 whenever $x_{i k}>0$. It follows that $n p_{k} \leq D_{k}^{-} V\left(x_{i}\right)$ whenever $x_{i k}>0$; consequently,

$$
a\left[n p \cdot x_{j}\right]^{a-1} n p_{k} x_{i k} \leq a\left[V\left(x_{i}\right)\right]^{a-1} D_{k}^{-} V\left(x_{i}\right) x_{i k} \text { for all } k .
$$

Summing this inequality over $k$ yields, for all $i \neq j$,

$$
\left[n p \cdot x_{j}\right]^{a-1} n p \cdot x_{i} \leq\left[V\left(x_{i}\right)\right]^{a-1} \nabla V\left(x_{i}\right) \cdot x_{i} .
$$

It follows from (5), (10), and $1 \geq V(x)$ that for all $i \neq j$,

$$
n p \cdot x_{i} \leq\left[n p \cdot x_{j}\right]^{1-a} .
$$

Because $a<1$, (8) and (11) imply

$$
n p \cdot x_{i} \leq 1,
$$

Because $\sum_{i=1}^{n} x_{i}=\mathbf{1}$ and $p \in \Delta_{m}$, summing (12) over all $i$ contradicts (8) and establishes the lemma.

Lemma 6.1 demonstrates that when $a>0$, the crucial lemma from Section 5 continues to hold. One can establish the same result for the Nash bargaining solution $(a=0)$ directly or through a limiting argument. Lemma 6.1 states that a player can obtain an allocation at least as good as a $1 / n$th share of each commodity according to his reported preferences, provided that his reported preferences are in an appropriate class. It would be interesting to find a condition on bargaining solutions that would imply the conclusion of Lemma 6.1.

Lemma 6.2. When $a<0, f(S, a)$ satisfies Axiom 4 (midpoint domination). 
Proof. Let $S \subset \mathbf{R}^{n}$ be a $0-1$ normalized compact convex set. When $a<0$, maximizing $W(\cdot, a)$ is equivalent to minimizing $\sum_{i=1}^{n} s_{i}^{a}$. Therefore, it suffices to show that if $s^{*}=\left(s_{1}^{*}, \ldots, s_{n}^{*}\right)$ solves

$$
\min \sum_{i=1}^{n} s_{i}^{a} \text { subject to } s \in S,
$$

then $s_{i}^{*} \geq 1 / n$ for all $i$. Without loss of generality, assume that $s_{1}^{*}=$ $\min \left\{s_{1}^{*}, \ldots, s_{n}^{*}\right\}$. Define the function $\varphi\left(s_{1}\right)=\max \left\{\lambda:\left(s_{1}, \lambda s_{-1}\right) \in S\right\}$. Because $S$ is $0-1$ normalized and convex, $\varphi$ is a real-valued, decreasing concave function satisfying $\varphi\left(s_{1}^{*}\right)=1, \varphi(1) \geq 0$, and $\left(s_{1}, \varphi\left(s_{1}\right) s_{-1}\right) \in S$ for all $s_{1} \in[0,1]$. Because $s^{*}$ solves (13), $s_{1}^{*}$ must solve

$$
\min \left\{s_{1}^{a}+\sum_{i=2}^{n}\left[\varphi\left(s_{1}\right) s_{i}\right]^{a}\right\} \text { subject to } s_{1} \in\left[s_{1}^{*}, 1\right] .
$$

Differentiating the objective function in (14) and using $a<0$, it follows that

$$
\left(s_{1}^{*}\right)^{a-1}+\varphi^{\prime}\left(s_{1}^{*}\right) \varphi\left(s_{1}^{*}\right)^{a-1} \sum_{i=2}^{n}\left(s_{1}^{*}\right)^{a} \leq 0,
$$

where $\varphi^{\prime}\left(s_{1}^{*}\right)=\lim _{h \rightarrow 0^{+}}\left[\varphi\left(s_{1}^{*}+h\right)-\varphi\left(s_{1}^{*}\right)\right] / h$. Because $\varphi(\cdot)$ is concave,

$$
\varphi^{\prime}\left(s_{1}\right) \geq \frac{\varphi(1)-\varphi\left(s_{1}\right)}{1-s_{1}} .
$$

Substituting (16) into (15) yields

$$
\left(1-s_{1}^{*}\right)\left(s_{1}^{*}\right)^{a-1}+\left[\varphi(1)-\varphi\left(s_{1}^{*}\right)\right] \varphi\left(s_{1}^{*}\right)^{a-1} \sum_{i=2}^{n}\left(s_{1}^{*}\right)^{a} \leq 0 .
$$

Because $\varphi\left(s_{1}^{*}\right)=1$ and $\varphi(1) \geq 0,(17)$ implies that $\left(s_{1}^{*}\right)^{a-1} \leq \sum_{i=1}^{n}\left(s_{i}^{*}\right)^{a}$ or

$$
\frac{1}{s_{1}^{*}} \leq \sum_{i=1}^{n}\left[\frac{s_{i}^{*}}{s_{1}^{*}}\right]^{a} \leq n,
$$

where the second inequality in (18) follows from $a<0$ and $s_{1}^{*}=$ $\min \left\{s_{1}^{*}, \ldots, s_{n}^{*}\right\}$.

As in the case of Lemma 6.1, Lemma 6.2 also holds for the Nash bargaining solution $(a=0)$. Combining Lemmas 6.1 and 6.2, one obtains a generalization of Theorem 5.1.

THEOREM 6.1. If $\left(p^{*}, x^{*}\right)$ is an EICE for the true preferences, then $x^{*}$ is a Nash equilibrium allocation for the distortion game determined by the solution $f(\cdot ; a)$. If $a \in[0,1]$, then it is an equilibrium strategy for all players to report the truncated linear utility function $L\left(\cdot ; p^{*}\right)$. If $a \leq 0$, then it is an equilibrium strategy for all players to report the linear utility function $U(y)=p^{*} \cdot y$. 
Proof. When $a \in[0,1]$, result follows from Lemma 6.1 and the same argument used to establish Theorem 5.1. When $a \leq 0$, the result follows from Lemma 6.2 and Sobel (1981).

The family of bargaining solutions shares the property that agents want to act as if the marginal unit that they are allocated is valuable. The optimal manipulation always involves claiming that the first units allocated add the minimum possible utility (subject to the concavity constraint). Strategies differ as to how agents should represent their utility for what they do not receive in equilibrium. Utilitarian solutions $(a>0)$ induce agents to claim that the units that they do not receive are worthless. Denying an interest in these units permits agents to exaggerate their interest for the units that they actually receive. Eqalitarian solutions $(a<0)$ favor an agent who appears to be getting a small utility relative to the maximum available utility for that agent. Consequently, it is in the interest of an agent faced with an egalitarian planner to report preferences in a way that exaggerate his maximum utility relative to the utility that he receives. This force creates an incentive to report linear preferences.

\section{REFERENCES}

Cao, X. (1982). "Preference Functions and Bargaining Solutions," in Proceedings of the 21st IEEE Conference on Decision and Control 1, 164-171.

Crawford, V., and Varian, H. (1979). "Distortion of Preferences and the Nash Theory of Bargaining," Econ. Letters 3, 203-206.

Dhillon, A., and Mertens, J.-F. (1999). "Relative Utilitarianism,” Econometrica 67, 471-498.

Hurwicz, L., Maskin, E., and Postlewaite, A. (1995). "Feasible Nash Implementation of Social Choice Rules When Designer Does Not Know Endowments or Production Sets," in The Economics of Informational Decentralization, (J. O. Ledyard, Ed.). Boston: Kluwer Academic Publishers.

Karni, E. (1998). "Impartiality: Definition and Representation," Econometrica 66, 1405-1415. Karni, E., and Safra, Z. (1996). "Individual Sense of Justice," Johns Hopkins University.

Kibris, O. (1998). "Misrepresentation of Utilities in Bargaining: Pure Exchange and Public Good Economies," Discussion paper, University of Rochester, Rochester.

Kurz, M. (1977). "Distortion of Preferences, Income Distribution, and the Case for a Linear Income Tax,” J. Econom. Theory 14, 291-298.

Kurz, M. (1980). "Income Distribution and Distortion of Preferences: The $l$-Commodity Case," J. Econom. Theory 22, 99-106.

Maskin, E. (1999). "Nash Implementation and Welfare Optimality," Rev. Econ. Stud. 66, 23-38.

Mas-Colell, A. (1980). "Remarks on the Game-Theoretic Analysis of a Simple Distribution of Surplus Problem," Int. J. Game Theory 9, 125-140.

Miyagawa, E. (1999). "Subgame-Perfect Implementation of Bargaining Solutions,” Discussion paper, University of Rochester, Rochester.

Nash, J. (1950). “The Bargaining Problem,” Econometrica 28, 155-162. 
Roth, A. (1979). Axiomatic Models of Bargaining. Berlin: Springer-Verlag.

Segal, U. (2000). "Let's Agree That All Dictatorships are Equally Bad," J. Polit. Econ. 108, 569-89.

Sobel, J. (1981). "Distortion of Utilities and the Bargaining Problem," Econometrica 49, 597-619.

Thomson, W. (1984). "The Manipulability of Resource Allocation Mechanisms," Rev. Econ. Stud. 51, 447-460.

Thomson, W. (1987). "The Vulnerability to Manipulative Behavior of Resource Allocation Mechanisms Designed to Select Equitable and Efficient Outcomes," in Information Incentives and Economic Mechanisms (T. Groves, R. Radner, and S. Reiter, Eds.). Minneapolis: University of Minnesota Press.

Thomson, W. (1988). "The Manipulation of the Shapley Value," Int. J. Game Theory 17, 101-127.

Thomson, W. (1994). "Cooperative Models of Bargaining," in Handbook of Game Theory, (R. Aumann and S. Hart, Eds.), Vol. II. Amsterdam: North-Holland. 